\title{
Design and Development of Perimeter Security Platform
}

\author{
Ramya Krishna Rajavolu, Venna Vijay Bharath Reddy
}

\begin{abstract}
There are many integrated perimeter security solutions available, the main objective of this paper is to provide cost effective solution. This paper mainly focuses on design of a Low-cost vibration module with RS485 interface, Controller hub with RS485 interface and Ethernet interface and a Command Centre server. The platform consists of RS485 daisy chained vibration sensor network connected to control server via Controller hub. It is designed to pin point the area of intrusion and cueing the camera to that specified location. It can be integrated with smart devices like PTZ/Thermal/IR cameras or radars. Each daisy chain consists of 250 sensors with each sensor 3 metres apart, each ethernet hub can handle 2 daisy chains. The Controller hub gets vibration sensor information via RS485 and transmits data to Command centre using TCP/IP protocol. The Controller centre identifies the location of the sensor and moves the PTZ camera to the specific location and live streams the data to the user.
\end{abstract}

Keywords:Low-cost vibration module with RS485 interface, Controller hub with RS485 interface and Ethernet interface and a Command Centre server.

\section{INTRODUCTION}

Starting late, Perimeter Security is used by Property owners and relationship of all sizes to safe guard their structures to be in threat to attacks from various social affairs of mental activists or blameworthy gatherings, as the issues become lucidly high and in that limit they ought to be guaranteed. To accomplish perception early, current information movement could be used. Such acknowledgment structures rely on current sensors and sensor systems with bleeding edge sensor-data examination and data mix. The sensors data offer capacities to help the structure managers in their work to supervise top level events and events executed by intruders and to shield the workplaces from outside ambushes [1]. To accomplish such systems it is essential to put a worthy degree of focal points on the starting events of the advancement, to delineate the customer requirements to the degree structure limits. To invigorate such systems they should be set up on a satisfying system structure. Along these lines, the probability to get vital system structure that give the most ideal approach to manage help treatment of scenes will development and help to avoid or if nothing else limit the deferred outcomes of attacks on essential establishment working conditions. To

pick the fitting structure design may be developed on the evaluation of partner needs through outline of get-togethers with different especially assigned space experts.

Revised Manuscript Received on December 5, 2019.

Ramya Krishna Rajavolu, Dept of ECE, Mallareddy Engineering College for Women, Secunderabad, India, ramyakrishna.71@gmail.com

Venna Vijay Bharath Reddy, Dept of ECE, Mallareddy Engineering College for Women, Secunderabad, India, vennavijayreddy@gmail.com

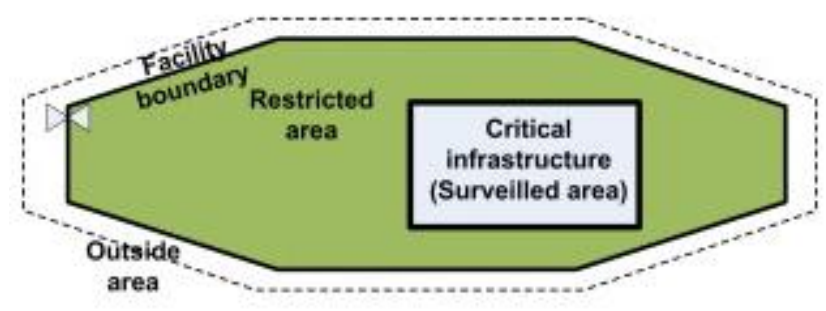

Fig 1. Perimeter to a restricted area

\section{SYSTEM MODULES}

The system comprises of 1) vibration sensor modules 2) Ethernet Hub and a 3) PC based control station. A group of 250 sensor modules are connected as RS485 daisy chain. The daisy chain is connected to the RS485 interface of Controller $\mathrm{Hub}$, whenever there is a vibration detected at a particular sensor module it transmits the data over RS485 to the ethernet hub, the hub then decodes the data and frames a TCP/IP packet and transmits to PC based control centre for further analysis.

\subsection{Vibration Sensor Module}

The vibration sensor module mainly consists of the following: PICmicrocontroller-PIC16LF88, Vibration Sensor - SQ SEN 200, Digital Transceiver-MAX3741EUA

The low power vibration sensor is connected to the microcontroller, the vibration, the vibrationsensor acts like a normally close switch which chatters close and open as it is tilted or vibrated. The microcontroller sends out the data through digital transceiver low power PIC microcontroller, vibration sensor and RS485 transceiver.

The PIC16LF88-I/SO is a PIC16 family 8-piece mind blowing (200 nanosecond heading execution) yet simple to-program (just 35 single word course) CMOS streak based Microcontroller packs surprising PIC®(RISC) structure. This thing offer each and every explanation behind social gathering of the well-seen mid-go x14 working with managed highlights including $7 \mathrm{kB}$ of addressable program memory size, 368bytes of information memory size, 16 all around noteworthy I/O pins, two comparators and 7-channel 10-piece simple to-bleeding edge (A/D) converter. This contraption works at a most marvelous rehash of $20 \mathrm{MHz}$ with wide working voltage of 2 to $5.5 \mathrm{~V}$.

The highlights unite 35 (14-piece wide) clear scrambling toward learn, 8 level mechanical assembly stack, 1 (9-piece) record select register, Hardware infringe in the wake of overseeing, 256bytes of EEPROM information memory, Power on reset (POR), Watch hound clock (WDT), Two 
8-piece pushed timekeepers, One 16-piece electronic clock, In-Circuit Serial Programming. In different applications like Industrial, Automotive, Consumer Electronics, Motor Drive and Control, Lighting, Medical, Security we can utilize this controller, at last we are utilizing it in edge security to shield foundation from interlopers.

The SQ-SEN-200 game-plan sensor goes about as a generally closed switch which chatters open and close as it is tilted or vibrated. Not the slightest bit like other ball-tube contraptions, the 200 is really an omnidirectional improvement sensor. The sensor will pass on evident on-off contact terminations while moving. Right when still, it generally speaking settles in a shut state. It is touchy to both tilt (static animating) and vibration (dynamic enlivening). This sign can be utilized to meddle with (wake up) a microcontroller or can be checked to think about the total and length of improvement. The sensor is completely separated, requires no sign trim, and can be utilized in a microcontroller burst in with circuit that draws as forlorn as $0.25 \mathrm{uA}$ of unwavering present as in fig2. The SQ-SEN-200 will undoubtedly be closedeven when the indisputable structure is incredibly still. Regardless, it is that $75 \%-95 \%$ of the time (subordinate upon course) when the sensor is still it will be shut. The virtuoso should structure the thing to search for high-to-low and low-to-high edge instigates rather than an open of shut condition of the switch.

\begin{tabular}{|l|l|l|l|}
\hline PARAMETER & MIN & MAX & UNITS \\
\hline Supply Voltage Range & 2.7 & 12 & Vdc \\
\hline Current Sink* & 0.00025 & 5 & $\mathrm{~mA}$ \\
\hline
\end{tabular}

Fig 2. Exciting characteristics

The electrical characteristics of sensor from above table shows that this sensor can take between $2.7 \mathrm{~V}$ and $12 \mathrm{~V}$, without affecting its sesing operation. When high voltages are applied , it will consume more current when the circuit is closed (as it usually is withthe sen-200). This will affect power conservation when using a battery. For this reason it is recommended to use a resistor and limit supplied voltage to the minimum $2.7 \mathrm{~V}$. I found that the usual $10 \mathrm{~K}$ does the trick when working with a $3 \mathrm{~V}$ coin-cell battery as power source

\section{COMMAND CENTER SERVER}

The Command Centre Server has an integrated sever software, which controls smart devices like PTZ camera, it also sends and receives data from the controller hub, it has a Graphical user interface which shows all the details about the perimeter. The user can configure the vibration module sensitivity based on his needs. The server also configures the controllerhub, the server GUI is developed using visual Studio.

The controller hub is based on CYCLONE IV Fpga, with LAN91C111 ethernet interface. The controller hub gets the health or vibration information form the sensor modules, It decodes the data and frames a TCP/IP packet which is sent to Command centre.

\section{iPIDS System Configuration}

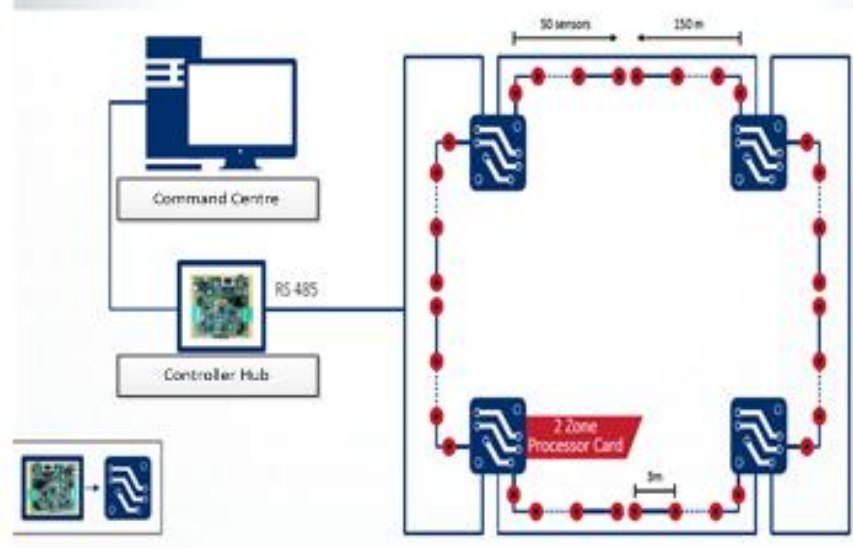

Fig 3. Schematic representation of Operation The controller Hub continuously scans the entire chain of sensor modules for health data and event data. In vibration sensor module the Vibration sensor is connected to a GPIO of pic micro controller, it produces pulses whenever there is an event or vibration, the micro controller counts the number of pulses and frames an encoded data packet with a unique id and transmits over RS485.

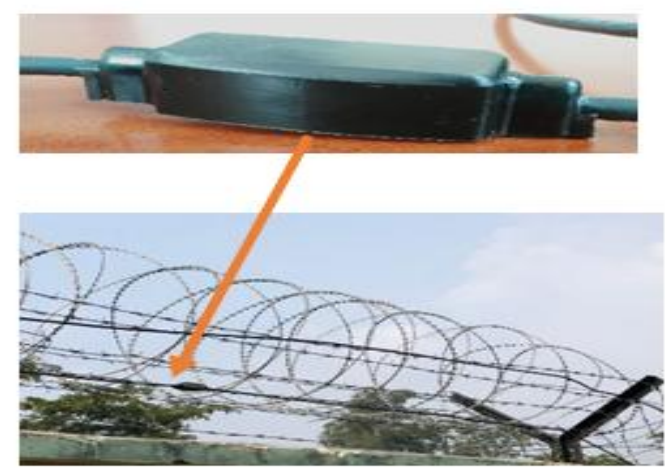

Fig 4. Position of System Design

The Controller Hub receives the event or health data and decodes the data and frames it into a TCP/IP data packet and transmits over Ethernet.

The command centre has a Graphical user Interface developed using visual studio. The GUI displays positions of sensor modules, intrusion detection and its health status. It has also an option to configure controller hub and vibration modules. 


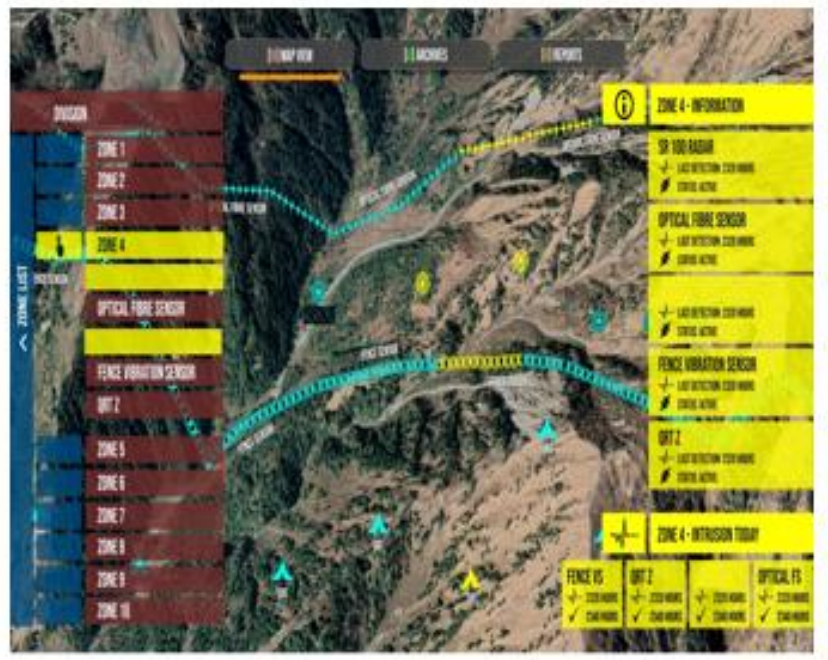

Fig 5. GUI Display

The GUI display in the above figure shows the positions of the vibration sensors placed, it also shows the time of intrusion, and it indicates the location of intrusion where it occurs. It also displays the health status of all the sensors.

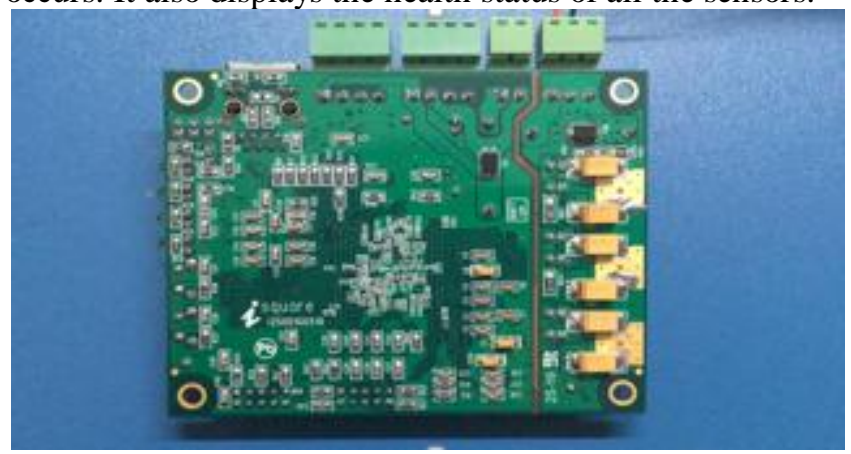

Fig 6. System Hardware

As the system hardware design shown in figure consists of PICmicrocontroller-PIC16LF88 and it will be interfaced with Vibration Sensor - SQ SEN 200, Digital Transceiver-MAX3741EUA to complete the design.

\section{CONCLUSION}

The main objective of this paper is to design a low cost perimeter surety platform which can be used in all conditions, it's easy to install and maintain. It can be used by defence forces, airport security and even in house hold security. It's has a very interactive Graphical User Interface to view the position of the sensors and to display the information due to intrusion detection. The GUI also controls the smart devices like PTZ camera to point towards the intruder. In future the Ethernet LAN cable can be replaced with wi-fi, and multiple types of sensors can be integrated. Right now it is applicable for airport and military defence applications and in future it will be widely used for house hold security.

\section{REFERENCES}

1. N. Hallberg, T. Timpka, H. ErikssonThe medical software quality deployment method Method Inform. Med, 38 (1999), pp. 66-73

2. F. Flammini, A. Gaglione, N. Mazzocca, V. Moscato, C. Pragliola

On-line integration and reasoning of multi- sensor data to enhance infrastructure surveillance J. Inform. Assur. Secur, 4 (2009), pp. 183-191

3. Integrated Perimeter Security System from 2007 IEEE Conference on Technologies for Homeland Security
4. A system design for surveillance systems protecting critical infrastructures from Journal of Visual Languages \& Computing Volume 25, Issue 6, December 2014, Pages 650-657

5. Intelligent video surveillance system from 2017 IEEE 23rd International Symposium for Design and Technology in Electronic Packaging (SIITME)

6. Airport Perimeter Security: Where we've been, Where we are, and Where we're going 2008 IEEE Conference on Technologies for Homeland Security

7. S. Shan, L. Wang, L. Li, Y. Chen An emergency response decision support system framework for application in e-government Inform. Technol. Manage, 13 (2012), pp. 411-427

8. E. Jungert, C. Grönwall, N. Hallberg, B. Kylesten, F. Lantz, L. Eriksson A generic architecture for surveillance systems Proceedings of theInternational Conference on Distributed Multimedia Systems (2010), pp. 57-63

9. F.J. Oppermann, S. Peter, Inferring technical constraints of a wireless sensor network application from end-user requirements", in: Proceedings of the 2010 6th International Conference on Mobile Adhoc and Sensor Networks (MSN) December 20-22, 2010, 168-175.

\section{AUTHORS PROFILE}

Ramya Krishna Rajavolu, Dept of ECE, Mallareddy Engineering College for Women, Secunderabad, India, ramyakrishna.71@ gmail.com

Venna Vijay Bharath Reddy, Dept of ECE, Mallareddy Engineering College for Women, Secunderabad, India, vennavijayreddy@gmail.com 